Davi de Araújo Brito Buttros' ${ }^{1}$

JORGE NAHAS-NETO ${ }^{2}$

Eliana Aguiar Petri Nahas ${ }^{2}$

LuCiana Mendes Cangussu ${ }^{1}$

Ana Beatriz Cesar Rodrigues BarRal ${ }^{1}$

MárCia Suemy KaWAKami ${ }^{3}$

Artigo original

Palavras-chave

Menopausa

Densidade óssea

Fatores de risco

Terapia de reposição hormonal

Keywords

Menopause

Bone Density

Risk factors

Hormone replacement therapy

\section{Fatores de risco para osteoporose em mulheres na pós-menopausa do sudeste brasileiro}

\author{
Risk factors for osteoporosis in postmenopausal women \\ from southeast Brazilian
}

\section{Resumo}

OBJETIVO: Avaliar a densidade mineral óssea (DMO) e os fatores de risco associados à osteoporose na pós-menopausa. MÉTODOS: Estudo clínico transversal com 431 mulheres (idade 40 - 75 anos). Foram incluídas mulheres com: amenorréia $>12$ meses e idade $\geq 45$ anos ou, ooforectomia bilateral $\geq 40$ anos, com DMO lescore T de coluna lombar/colo de fêmur) pelo DEXA dos últimos 12 meses. Fatores de risco avaliados: idade, idade e tempo de menopausa, tabagismo, atividade física (30 min/5 vezes/ semana), artrite reumatoide (AR), uso de corticoterapia e de terapia hormonal (TH), fratura prévia, fratura materna de quadril e índice de massa corpórea $(I M C=$ peso/altura²). Foram empregodos teste do $\chi^{2}$ e método de regressão logística no risco (Odds Ratio - OR) para osteoporose. RESULTADOS: Pelos critérios da Organização Mundial da Saúde, 106 (24,6\%) mulheres apresentavam osteoporose (escore T s-2,5DP), 188 (43,6\%) osteopenia $(-1,0 /-2,4 \mathrm{DP})$ e $137(31,8 \%)$ eram normais ( $\geq-1, \mathrm{ODP})$. Foi detectada osteoporose em $12 \%$ das mulheres com idade entre 40 e 49anos, em $21,8 \%$ no grupo de 50 a 59 anos e 45,7\% nas mulheres com idade $>60$ anos ( $p<0,001)$. Osteoporose ocorreu em $11,8 \%$ com tempo de menopausa $<5$ anos, 29,4\% de $6-10$ anos, e $41 \%$ $>10$ anos $(p<0,001)$. Naquelas com idade da menopausa $<40$ anos, $80 \%$ apresentaram osteopenia/osteoporose $(p=0,03)$ e com $I M C<20 \mathrm{~kg} / \mathrm{m}^{2}, 50 \%$ osteoporóticas $(p<0,001)$. $\bigcirc$ risco de detectar osteoporose aumentou com a idade $\mid O R=1,1$; IC95\%=1,0-1,1), tempo de menopausa $(O R=1,0$; IC $95 \%=1,0-1,1)$, tabagismo atual $\mid(O R=2,1$; IC95\%=1,2-3,8), AR $(O R=3,6$; IC95\%=1,3-9,6) e história materna de fratura de quadril $(O R=2,1 ; \mid C 95 \%=1,1-3,0)$ $(\mathrm{p}<0,05)$. Contrariamente, o uso de TH $(\mathrm{OR}=0,49 ; \mathrm{IC} 95 \%=0,3-0,9)$ e elevado IMC $(\mathrm{OR}=0,9 ; \mid \mathrm{IC} 95 \%=0,8-0,9)$ reduziram o risco de detecção da osteoporose $(p<0,05)$. CONCLUSÃO: Em mulheres na pós-menopausa, idade, tempo de menopausa, tabagismo e história materna de fratura, foram indicadores clínicos do risco para osteoporose, enquanto o uso de TH e IMC elevado, apresentaram-se como fatores protetores.

\section{Abstract}

PURPOSE: To evaluate bone mineral density (BMD) and their risk factors associated with postmenopausal osteoporosis. METHODS: A cross-sectional clinical study was performed on 431 women (aged 40 - 75 years). Inclusion criteria: amenorrhea $>12$ months and age $\geq 45$ years or, bilateral oophorectomy $\geq 40$ years with BMD values (T-score of lumbar spine/femur neck) by DXA of the last 12 months. Risk factors evaluated: age, age and time of menopause, smoking, physical activity (30 min/5 times/week), rheumatoid arthritis (RA), use of corticotherapy and hormone therapy (HT), previous fracture, maternal hip fracture and body mass index (BMl=weight/height $\left.{ }^{2}\right)$. The $\chi^{2}$ test and the logistic regression method (Odds Ratio - OR) were used to determine osteoporosis risk. RESULTS: According to WHO criteria, 106 (24.6\%) women showed osteoporosis (T-score s-2.5 DP), 188 (43.6\%) osteopenia (-1.0/-2.4 DP), and 137 (31.8\%) were normal ( $\geq-1.0$ DP). Osteoporosis was detected in $12 \%$ of women aged $40-49$ years, in $21.8 \%$ of women aged $50-59$ years and in $45.7 \%$ of women aged $>60$ years $(p<0.001)$. Osteoporosis occurred in $11.8 \%$ of women with a menopause period $<5$ years, in $29.4 \%$ with a menopause period from 6 to 10 years, and in $41 \%$ of women with a menopause period $>10$ years $(p<0.001)$. Of the women with early menopause, $80 \%$ showed osteopenia/ osteoporosis ( $\mathrm{p}=0.03$ ), and of those with $\mathrm{BMl}<20 \mathrm{~kg} / \mathrm{m}^{2}, 50 \%$ were osteoporotic ( $\mathrm{p}<0.001$ ). The risk for osteoporosis
Correspondência:

Eliana Aguiar Petri Nahas Departamento de Ginecologia e Obstetrícia - Faculdade de Medicina de Botucatu - Universidade Estadual Paulista - UNESP Distrito de Rubiōo Júnior $s / n$ (EP: 18650-000 - Botucatu (SP), Brasi Tel.: 14 3811-6227/ 14 3841-7193 - Fax: 14 3882-1933

Recebido $31 / 05 / 2011$
Setor de Climatério e Menopausa do Departamento de Ginecologia e Obstetrícia da Faculdade de Medicina de Botucatu da Universidade Estadual Paulista "Júlio de Mesquita Filho" - UNESP - Botucatu (SP), Brasil.

' Pós-graduando do Programa de Pós-Graduação em Ginecologia, Obstetrícia e Mastologia da Faculdade de Medicina de Botucatu da Universidade Estadual Paulista "Júlio de Mesquita Filho" - UNESP - Botucatu (SP), Brasil.

2 Professores Doutores do Departamento de Ginecologia e Obstetrícia da Faculdade de Medicina de Botucatu da Universidade Estadual Paulista "Júlio de Mesquita Filho"- UNESP - Botucatu (SP), Brasil.

${ }^{3}$ Acadêmica de Medicina da Faculdade de Medicina de Botucatu da Universidade Estadual Paulisła "Júlio de Mesquita Filho" - UNESP - Botucatu (SP), Brasil; Bolsista de Iniciação Científica da Fundação de Amparo à Pesquisa do Estado de São Paulo - FAPESP Processo n 2008/10881-6. 


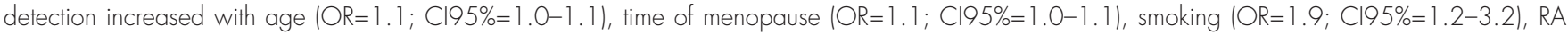
$(O R=3.6 ; C 195 \%=1.3-9.6)$ and maternal fracture history $(O R=2.1 ; C l 95 \%=1.1-3.0)(p<0.05)$. In contrast, $\mathrm{HT}$ use $(\mathrm{OR}=0.3 ; 95 \% \mathrm{Cl}=0.2-0.6)$ and high BMI $(O R=0.9 ; 95 \% \mathrm{Cl}=0.8-0.9)$ reduced the risk ( $p<0.05)$. CONCLUSION: In postmenopausal women, age, time of menopause, smoking and maternal history of fracture were clinical indicators of risk for osteoporosis, whereas $\mathrm{HT}$ use and high BMI proved to be protective factors.

\section{Introdução}

O aumento na expectativa de vida proporcionou crescimento acentuado da população mais velha, tanto nos países desenvolvidos como naqueles em desenvolvimento. No Brasil, em 2010, estima-se que havia 14 milhões de idosos, dos quais mais da metade é composto por mulheres $(55,8 \%)^{1}$. Assim, com o aumento da sobrevida, se eleva a ocorrência de doenças relacionadas ao envelhecimento da mulher ${ }^{2}$. Entre essas doenças destaca-se a osteoporose. A Organização Mundial de Saúde (OMS) define a osteoporose como a redução da massa óssea associada a desarranjo da microarquiterura óssea, que resulta em maior fragilidade óssea e risco aumentado de fraturas ${ }^{3}$. Nos primeiros anos pós-menopausa, em reposta ao hipoestrogenismo, ocorre rápida perda de massa óssea ${ }^{4}$. O declínio na densidade mineral óssea (DMO) e na integridade estrutural resulta em aumento do risco para osteoporose em mulheres ${ }^{5}$. A principal consequência clínica é a fratura osteoporótica, que ocorre principalmente em colo de fêmur, vértebra e punho². Em 2009, foram registrados 1478 óbitos por fratura de fêmur segundo o Ministério da Saúde 6 . A dificuldade do retorno às atividades diárias, os resultados funcionais ruins nos pós-operatórios e a alta taxa de dependência levam à queda significativa da qualidade de vida ${ }^{7}$.

Em 1994, a OMS definiu critério para o diagnóstico de osteoporose baseado na mensuração da DMO da coluna e do quadril por meio do dual energy $x$-ray absorptiometry (DEXA), que são reportados em T-score, expresso em unidades de desvio padrão (DP). Este é baseado em indivíduos do mesmo sexo, raça e etnia, comparando a DMO de mulheres na pós-menopausa com a de adultos jovens $(20-29 \text { anos })^{3}$. A relação entre o escore $\mathrm{T}$ e o risco de fratura não tem sido sistematicamente avaliada ${ }^{8}$. Muitas mulheres, na pós-menopausa com fraturas, não apresentam DMO consistente com osteoporose baseados no critério diagnóstico da OMS. No estudo National Osteoporosis Risk Assesment (NORA) ${ }^{9}$, publicado em 2001, envolvendo 200.160 mil mulheres americanas na pós-menopausa ( $\geq 50$ anos), observou-se que entre aquelas que sofreram fraturas por fragilidade, apenas $18 \%$ foram classificadas como osteoporóticas 9 . Portanto, se a conduta terapêutica for baseada somente na DMO, cerca de metade das mulheres na pós-menopausa não seriam tratadas antes de ocorrer a primeira fratura ${ }^{10}$.

Apesar de a DMO ser muito importante, é apenas um dos componentes na análise do risco para fraturas, pois 60 a $70 \%$ da resistência do osso se devem à quantidade óssea que é medida pela densitometria e o restante se relaciona à qualidade óssea que não pode ser avaliada adequadamente ${ }^{11,12}$. Outros fatores como idade, história familiar de fratura, baixo índice de massa corpórea, fratura prévia por fragilidade, também são componentes para o risco de fraturas ${ }^{8,13}$. O risco de uma mulher de 50 anos ou mais apresentar uma fratura ao longo da vida é de mais de $40 \%{ }^{14}$. Mulheres na peri ou pós-menopausa que tiveram fratura, têm aproximadamente duas vezes aumentado o risco de outra fratura ${ }^{15}$. História familiar de fraturas também aumenta significativamente o risco, pois o pico de massa óssea de uma mulher é determinada geneticamente. Estudos sugerem que aproximadamente $80 \%$ dos casos de baixa massa óssea podem ser atribuídos a fatores genéticos ${ }^{16}$.

De acordo com a North American Menopause Society $(\mathrm{NAMS})^{8}$ e do National Osteoporosis Foundation (NOF) ${ }^{17}$, todas as mulheres na pós-menopausa deveriam ser avaliadas quanto aos fatores de risco para osteoporose e fraturas, pois atualmente o tratamento medicamentoso é indicado na associação dos valores da DMO e fatores de risco. Assim, a avaliação dos fatores associados à osteoporose e à fratura é de suma importância na abordagem de mulheres na pósmenopausa, buscando identificar as pacientes de risco para osteoporose com a instituição de medidas terapêuticas e preventivas e consequente melhoria da qualidade de vida desta faixa da população.

Baseado nesses dados, o objetivo do presente estudo foi avaliar a DMO e identificar os fatores de risco associados à osteoporose em mulheres na pós-menopausa.

\section{Métodos}

\section{Desenho do estudo e seleção da amostra}

Trata-se de estudo clínico, analítico e transversal. O grupo populacional foi constituído de 431 mulheres atendidas no Ambulatório de Climatério e Menopausa da Faculdade de Medicina de Botucatu, de janeiro a dezembro de 2010. A amostra foi não-probabilística e por adesão. $\mathrm{O}$ tamanho amostral foi baseado no número médio do atendimento ambulatorial anual, que preenchesse os critérios propostos pelo estudo. Foram incluídas no protocolo mulheres com: data da última menstruação há pelo menos 12 meses e idade $\geq 45$ anos; ooforectomia bilateral com idade $\geq 40$ anos; valores de densitometria óssea nos últimos 12 meses. Foram excluídas mulheres com elevado 
grau de osteoartrose pela alteração na DMO. Assim, das 556 consultas médicas realizadas em 2010, 125 pacientes foram excluídas por dados incompletos, perimenopausa, idade $<45$ anos e não ooforectomizada e osteoartrose acentuada; sendo que 431 mulheres foram elegíveis para o estudo. Foram esclarecidos para as pacientes selecionadas, os objetivos e procedimentos aos quais seriam submetidas, e solicitadas às assinaturas do termo de consentimento livre e esclarecido (TCLE), exigência da Resolução $\mathrm{n}^{\circ}$ 196/1996 do Conselho Nacional de Saúde. O projeto de pesquisa foi encaminhado ao Comitê de Ética em Pesquisa da Faculdade de Medicina de Botucatu da Universidade Estadual Paulista, recebendo parecer favorável.

\section{Metodologia}

Inicialmente, no dia da consulta, foram coletados, por meio de entrevista, os dados das 431 participantes. Foram obtidos: idade, idade da menopausa, tempo de menopausa, tabagismo (fumo atual independentemente do número de cigarros), consumo de álcool (>duas doses/dia), uso ou não de terapia hormonal, doenças gastrointestinais acompanhadas de má-absorção, artrite reumatóide, doenças da tireóide, uso de corticoterapia crônica, história pessoal de fratura por fragilidade (quadril, vertebral, punho), antecedente materno de fratura de quadril e atividade física. Foram consideradas ativas as mulheres que praticavam exercícios físicos regulares por 30 minutos pelo menos três vezes na semana.

\section{Avaliação antropométrica}

Foram obtidas as seguintes informações para avaliação antropométrica: peso, altura e índice de massa corpórea $\left(\mathrm{IMC}=\right.$ peso/altura $\left.{ }^{2}\right)$. Para mensuração do peso, utilizou-se a balança antropométrica tipo plataforma, graduada a cada 100 gramas, com capacidade até $150 \mathrm{~kg}$, com precisão de $0,1 \mathrm{~kg}$, com a paciente descalça e com mínimo de roupa. A estatura foi determinada em antropômetro portátil afixado em parede, com precisão de $0,1 \mathrm{~cm}$. Foram empregados os critérios da World Health Organization de 2002 para classificação das pacientes, conforme o IMC: menor que $18,5 \mathrm{~kg} / \mathrm{m}^{2}$ como baixo peso, de 18,5 a $24,9 \mathrm{~kg} / \mathrm{m}^{2}$ normal, de 25 a $29,9 \mathrm{~kg} / \mathrm{m}^{2}$ sobrepeso, de 30 a $34,9 \mathrm{~kg} /$ $\mathrm{m}^{2}$ obesidade grau I, de 35 a $39,9 \mathrm{~kg} / \mathrm{m}^{2}$ obesidade grau II e maior ou igual a $40 \mathrm{~kg} / \mathrm{m}^{2}$ obesidade grau III.

\section{Densidade mineral óssea}

Quanto à avaliação da DMO, foram obtidos os laudos de densitometria óssea dos últimos 12 meses realizados pelo Setor de Radiologia da Faculdade de Medicina de Botucatu. A DMO foi medida por meio do exame de DEXA em coluna lombar (L1 a L4) e colo de fêmur, pelo aparelho Hologic QDR-2000 (Waltham, MA, EUA). A técnica baseia-se na atenuação, pelo corpo do paciente, de um feixe de radiação gerado por uma fonte de raio- $\mathrm{X}$ com dois níveis de energia. Os coeficientes de variação no período dos exames foram inferiores a $2 \%$. Os valores foram classificados pelo escore $\mathrm{T}$ que corresponde ao valor de DMO média de mulheres jovens normais menos a DMO da paciente, dividido pelo DP da média de jovens normais. As participantes foram divididas de acordo com o valor de escore $\mathrm{T}$ em: normal quando para valor maior ou igual -1,0 DP; ostopenia, valores entre -1,0 e -2,4 DP; e osteoporose valor igual ou menor que $-2,5 \mathrm{DP}$.

\section{Avaliação laboratorial}

Para avaliação laboratorial foram empregadas as dosagens de cálcio sérico, fosfatase alcalina (FA) e calciúria de 24 horas. A partir destes dados, foram preenchidos os protocolos com os valores laboratoriais. Todas as avaliações laboratoriais foram realizadas pelo Laboratório de Análises Clínicas da Faculdade de Medicina de Botucatu. As mensurações foram processadas por analisador bioquímico automático (Vitros Fusion $5.1^{\circledR}$ ), sendo quantificados pelo método da química seca, utilizando-se reagentes comerciais específicos (Jonhson-Jonhson ${ }^{\circledR}$ ). Os valores considerados dentro da normalidade para o cálcio sérico foram de 8,4 a 10,2 mg/dL, para a FA de 36 a $129 \mathrm{U} / \mathrm{L}$ e para calciúria, valor inferior a $250 \mathrm{mg} / 24$ horas.

\section{Análise estatística}

A partir dos dados foi construída tabela contendo as características clínicas, antropométricas e laboratoriais das pacientes. Todos estes dados foram apresentados em forma de média \pm DP para as diversas variáveis. Para isto, foi realizada uma análise descritiva. A homogeneidade entre os grupos segundo a densidade mineral óssea (normal, osteopenia e osteoporose), em relação às características sociodemográficas, clínicas e antropométricas foi avaliada pelo teste do $\chi^{2}$ ou exato de Fisher de acordo com o parâmetro analisado (características categóricas ou qualitativas). Para análise dos dados, os valores foram expressos em número e porcentagem entre parênteses.

Foi realizada análise multivariada por regressão logística binária, considerando-se um nível de significância $\mathrm{p}<0,05$ e intervalo de confiança (IC) de $95 \%$, com cálculo da respectiva Odds Ratio (OR), para se observar as possíveis associações existentes entre o risco de osteoporose (variável dependente) e as variáveis influentes do risco (variáveis independentes). Foram testadas todas as variáveis clínicas (idade, idade e tempo de menopausa, IMC, exercício físico, tabagismo, terapia hormonal, fratura prévia, história materna de fratura de quadril, corticoterapia crônica e artrite reumatóide) e laboratoriais (cálcio, FA e calciúria de 24h) através do ajuste do modelo de regressão logística múltipla utilizando-se procedimento stepwise para as variáveis que 
apresentaram diferença significativa. Apenas os resultados estatisticamente significativos foram apresentados.

Os testes estatísticos foram bilaterais e o nível de significância adotado foi de 5\%. As análises foram realizadas utilizando-se Statistical Analyses System (SAS), versão 9.2, pelo Grupo de Apoio à Pesquisa (GAP) da Faculdade de Medicina de Botucatu que deu o atendimento metodológico e conduziu os procedimentos estatísticos.

\section{Resultados}

As características descritivas clínicas e densitométricas das 431 mulheres na pós-menopausa estão apresentadas na Tabela 1 . Observa-se que a média de idade foi $54,1 \pm 6,9$ anos com tempo de menopausa de 7,5 $\pm 5,8$ anos. As pacientes foram classificadas em média como sobrepeso (IMC de 25 a $29,9 \mathrm{~kg} / \mathrm{m}^{2}$ ). Pelos resultados da densitometria óssea, apresentavam-se em média com

Tabela 1. Características descritivas das 431 mulheres na pós-menopausa

\begin{tabular}{lccc}
\hline Variáveis & Média \pm DP & Mínimo & Maximo \\
\hline Idade (anos) & $54,1 \pm 6,9$ & 40,0 & 75,0 \\
Idade da menopausa (anos) & $48,4 \pm 5,3$ & 33,0 & 56,0 \\
Tempo menopausa (anos) & $7,5 \pm 5,8$ & 1,0 & 28,0 \\
IMC (kg/m²) & $28,2 \pm 5,3$ & 16,8 & 42,7 \\
Cálcio sérico (mg/dL) & $9,5 \pm 0,5$ & 8,0 & 12,0 \\
Fosfatase alcalina (U/L) & $77,7 \pm 22,4$ & 37,0 & 151,0 \\
Calciúria (mg/24 h) & $144,8 \pm 78,3$ & 42,8 & 379,7 \\
DM0 coluna lombar (DP) & $-1,3 \pm 1,2$ & $-4,2$ & 2,4 \\
DM0 colo de fêmur (DP) & $-1,0 \pm 1,2$ & $-4,0$ & 2,0 \\
\hline
\end{tabular}

IMC: índice de massa corpórea; DMO: densidade mineral óssea; DP: desvio padrão. osteopenia em coluna lombar e colo de fêmur. Os valores médios de cálcio, FA e calciúria encontravam-se dentro dos parâmetros da normalidade (Tabela 1).

Características clínicas das pacientes divididas segundo a densidade mineral óssea em normal, osteopenia e osteoporose, foram submetidas à comparação estatística, e estão representadas nas Tabelas 2 e 3. Pelos critérios da OMS, $106(24,6 \%)$ mulheres apresentavam osteoporose (escore $\mathrm{T} \leq-2,5 \mathrm{DP}), 188$ (43,6\%) osteopenia (-1,0 a -2,4 DP) e $137(31,8 \%)$ tinham DMO normal ( $\geq-1,0$ DP). Foi detectada osteoporose em $12 \%$ das mulheres com idade entre 40 e 49 anos, $21,8 \%$ entre 50 e 59 anos e $45,7 \%$ acima de 60 anos $(\mathrm{p}<0,001)$. Osteoporose ocorreu em $11,8 \%$ das pacientes com tempo de menopausa abaixo de 5 anos, 29,4\% de 6 a 10 anos, e $41 \%$ quando acima de 10 anos $(\mathrm{p}<0,001)$. Naquelas com menopausa precoce, $80 \%$ apresentaram osteopenia ou osteoporose $(\mathrm{p}=0,032)$ e com IMC menor que $20 \mathrm{~kg} / \mathrm{m}^{2}, 50 \%$ eram osteoporóticas $(\mathrm{p}<0,001)$ (Tabela 2).

Em relação à ocorrência de algumas características clínicas, foi encontrado que 35,9\% eram usuárias de TH, $27,3 \%$ realizavam caminhadas regulares pelo menos três vezes na semana; $23,8 \%$ eram tabagistas; $18,1 \%$ relatavam idade da menopausa abaixo de 40 anos; $11,8 \%$ referiam fratura; $10,7 \%$ apresentavam história materna de fratura de quadril; 4,8\% eram usuárias de corticoterapia crônica e 4,0\% tinham AR. Na associação entre essas características clínicas e o perfil da DMO, foi observada diferença significativa na distribuição percentual quanto ao tabagismo, fratura prévia, AR e fratura materna de quadril, com maior ocorrência entre as mulheres com osteoporose $(\mathrm{p}<0,05)$. Não houve associação com a atividade física e

Tabela 2. Associaccão entre as características clínicas categóricas e o perfil de densidade mineral óssea (normal, osteopenia e osteoporose) das 431 mulheres

\begin{tabular}{|c|c|c|c|c|c|c|c|c|}
\hline \multirow[t]{3}{*}{ Características clínicas } & \multirow[t]{3}{*}{ n } & \multicolumn{2}{|c|}{ Normal } & \multicolumn{2}{|c|}{ Osteopenia } & \multicolumn{2}{|c|}{ Osteoporose } & \multirow[t]{3}{*}{ Valor p* } \\
\hline & & n & $\%$ & n & $\%$ & n & $\%$ & \\
\hline & & 137 & 31,8 & 188 & 43,6 & 106 & 24.6 & \\
\hline Faixa efária (anos) & & & & & & & & $<0,001$ \\
\hline $40-49$ & 108 & 43 & 39,9 & 52 & 48,1 & 13 & 12,0 & \\
\hline $50-59$ & 229 & 81 & 35,4 & 98 & 42,8 & 50 & 21,8 & \\
\hline$\geq 60$ & 94 & 13 & 13,8 & 38 & 40,4 & 43 & 45,7 & \\
\hline$\geq 41$ & 353 & 120 & 34,0 & 144 & 40,8 & 89 & 25,2 & \\
\hline Tempo menopausa (anos) & & & & & & & & $<0,001$ \\
\hline$\leq 5$ & 195 & 84 & 43,1 & 88 & 45,1 & 23 & 11,8 & \\
\hline $6-10$ & 119 & 35 & 29,4 & 49 & 41,2 & 35 & 29,4 & \\
\hline$>10$ & 117 & 18 & 15,4 & 51 & 43,6 & 48 & 41,0 & \\
\hline $25-29,9$ & 164 & 49 & 29,9 & 77 & 47,0 & 38 & 23,2 & \\
\hline$\geq 30$ & 151 & 63 & 41,7 & 63 & 41,7 & 25 & 16,6 & \\
\hline
\end{tabular}

Valor expresso em número e porcentagem; IMC: índice de massa corpórea; * diferença estatística entre os grupos se $p<0,05$ (teste do $\chi^{2}$ ). 
Tabela 3. Associação entre as características clínicas qualitativas e o perfil de densidade mineral óssea (normal, osteopenia e osteoporose) das 431 mulheres

\begin{tabular}{|c|c|c|c|c|c|c|c|c|}
\hline \multirow[t]{3}{*}{ Características clínicas } & \multirow[t]{3}{*}{ n } & \multicolumn{2}{|c|}{ Normal } & \multicolumn{2}{|c|}{ Osteopenia } & \multicolumn{2}{|c|}{ Osteoporose } & \multirow[t]{3}{*}{ Valor $p$} \\
\hline & & n & $\%$ & n & $\%$ & $n$ & $\%$ & \\
\hline & & 137 & 31,8 & 188 & 43,6 & 106 & 24,6 & \\
\hline Tabagismo atual & & & & & & & & $<0,05^{\star}$ \\
\hline Sim & 103 & 22 & 21,4 & 45 & 43,7 & 36 & 35,0 & \\
\hline Não & 328 & 115 & 35,1 & 143 & 43,6 & 70 & 21,3 & \\
\hline Fratura prévia & & & & & & & & $0,01^{\star}$ \\
\hline Não & 380 & 124 & 32,6 & 167 & 43,9 & 89 & 23,4 & \\
\hline Artrite reumatóide & & & & & & & & $0,02^{\star \star}$ \\
\hline Sim & 17 & 2 & 11,8 & 6 & 35,3 & 9 & 52,9 & \\
\hline Não & 414 & 135 & 32,6 & 182 & 44,0 & 97 & 23,4 & \\
\hline Uso de terapia hormonal & & & & & & & & $<0,001^{\star}$ \\
\hline Não & 384 & 129 & 33,6 & 167 & 43,5 & 88 & 22,9 & \\
\hline
\end{tabular}

Valor expresso em número e porcentagem; * diferença estatística entre os grupos se $p<0,05$ (teste do $\chi^{2}$ ); * * diferença estatística entre os grupos se $p<0,05$ (teste exato de Fisher).

uso de corticoterapia. Em relação ao uso de TH, a maior porcentagem das usuárias apresentava DMO normal ou osteopenia (39,4 e 46,5\%, respectivamente); por outro lado, na maioria das não usuárias predominava osteopenia ou osteoporose ( 42 e $30,4 \%$, respectivamente, $\mathrm{p}<0,001$ ) (Tabela 3). Nenhuma associação foi observada entre DMO e valores de cálcio $(\mathrm{p}=0,174), \mathrm{FA}(\mathrm{p}=0,901)$ e calciúria $(\mathrm{p}=0,759)-$ dados não apresentados.

Avaliando o risco para osteoporose na presença das variáveis influentes, foi encontrado que o risco aumentou com a idade $(\mathrm{OR}=1,06$; IC95\%=1,0-1,1), tempo de menopausa $(\mathrm{OR}=1,0 ; \mathrm{IC} 95 \%=1,0-1,1)$, tabagismo atual $(\mathrm{OR}=2,1 ; \mathrm{IC} 95 \%=1,2-3,8), \mathrm{AR}(\mathrm{OR}=3,6$; IC95\% $=1,3-$ $9,6)$ e história materna de fratura de quadril $(\mathrm{OR}=2,1$; IC95\% $=1,1-3,0)(\mathrm{p}<0,05)$. Em contraste, o uso de TH $(\mathrm{OR}=0,5 ; \mathrm{IC} 95 \%=0,3-0,9)$ e elevado IMC $(\mathrm{OR}=0,9$; IC95\% $=0,8-0,9)$ associaram-se com significante redução do risco para osteoporose (Tabela 4). Demais variáveis clínicas e antropométricas não influenciaram significativamente no risco de osteoporose.

\section{Discussão}

A prevalência de osteoporose e da osteopenia na população estudada são comparáveis com os dados da Third National Health and Nutrition Examination Survey que indicam que aproximadamente $18 \%$ da população feminina americana com mais de 50 anos tem osteoporose e 38 a $50 \%$ apresenta osteopenia ${ }^{8}$. A OMS estima que uma em cada seis mulheres com 50 anos apresentam osteoporose de quadril, prevalência que aumenta para $50 \%$ aos 80 anos $^{7}$. O aumento da prevalência com a idade também
Tabela 4. Análise multivariada em funcão das variáveis clínicas influentes no risco para osteoporose nas 431 mulheres na pós-menopausa

\begin{tabular}{lccc}
\hline Variáveis & Odds Ratio (OR)* & IC95\% & Valor p** \\
\hline Idade (anos) & 1,1 & $1,0-1,1$ & 0,03 \\
\hline Tempo menopausa (anos) & 1,1 & $1,0-1,2$ & 0,04 \\
Tabagismo atual & 2,2 & $1,2-3,8$ & 0,01 \\
Uso de terapia hormonal & 0,5 & $0,2-0,9$ & 0,02 \\
IMC & 0,8 & $0,7-0,9$ & $<0,001$ \\
Artrite reumatóide & 3,6 & $1,4-9,6$ & 0,01 \\
Frafura materna de quadril & 2,2 & $1,2-4,1$ & 0,02 \\
\hline
\end{tabular}

IC: intervalo de confiança; *apresentados apenas os resultados estatisticamente significativos; demais variáveis não mostraram significância; * $p<0,05$ (regressão logística).

foi demonstrado em nosso estudo. Entre as pacientes com mais de 60 anos, aproximadamente $45 \%$ apresentavam osteoporose e apenas $21,8 \%$ das mulheres com idade entre 50 e 59 anos eram osteoporóticas.

O hipoestrogenismo é considerado importante fator de risco para baixa densidade mineral óssea ${ }^{4,18}$. Em recente estudo, Demir et al. ${ }^{19}$ estudaram 2.769 mulheres na pósmenopausa, correlacionando a ocorrência de osteoporose com o tempo de menopausa. Demonstraram que 10,6\% das pacientes com até três anos de pós-menopausa tinham osteoporose contra $32,9 \%$ das pacientes com sete anos ou mais. Os autores discutem que a redução da DMO destas pacientes também pode ser atribuída pelos fatores relacionados ao envelhecimento. Acredita-se que a deficiência estrogênica seja responsável por dois terços da perda de massa óssea ${ }^{8}$. Em concordância com estudos prévios, nossos dados também demonstram o hipoestrogenismo como importante fator de risco. Observamos que apenas $12 \%$ das pacientes com até cinco anos de menopausa têm 
o diagnóstico de osteoporose em comparação com $41 \%$ das pacientes com mais de dez anos de menopausa.

O mesmo raciocínio é aplicado ao uso de terapia hormonal para a prevenção da osteoporose. Neste estudo, foi observado efeito de redução no risco para baixa DMO entre as usuárias de $\mathrm{TH}$, que correspondiam a 35,9\% das participantes. Os efeitos benéficos da terapia estrogênica sobre a preservação da DMO e redução do risco de fraturas estão bem estabelecidos ${ }^{8,20}$. Em 2002, uma metanálise avaliando 57 estudos randomizados, placebo-controlados encontrou consistente aumento de DMO em todos os sítios entre usuárias de $\mathrm{TH}^{21}$. O estudo Women's Health Iniciative (WHI $)^{22}$ avaliou os riscos e benefícios da $\mathrm{TH}$ em mulheres na pós-menopausa, com idade entre 50 e 79 anos. Tratou-se de estudo randomizado, duplo-cego, placebo-controlado, com 8.506 mulheres tratadas com estrogênios equinos conjugados (EEC 0,625 mg) associados ao acetato de medroxiprogesterona (AMP 2,5 mg), continuamente, e 8.102 mulheres sob placebo (grupo controle). No seguimento de cinco anos, observou-se entre as usuárias de TH significante ganho de DMO de 4,5\% em coluna e de 3,7\% em fêmur total, quando comparadas ao grupo placebo ${ }^{22}$. Evidências tanto de estudos controlados como observacionais indicam que a $\mathrm{TH}$ reduz o risco de fraturas osteoporóticas em mulheres na pós-menopausa. O estudo NORA ${ }^{9}$, envolvendo 200.160 mulheres na pós-menopausa, relatou que o uso atual de estrogênio terapia associou-se com significante redução do risco de nova fratura ${ }^{9}$. Este resultado foi confirmado pelo WHI, que demonstrou significante redução do risco de fraturas de quadril, vértebras e corpo total entre usuárias de TH quando comparado ao placebo ${ }^{22,23}$.

É conhecido o fator genético no risco para osteoporose. No presente estudo, o antecedente familiar de fratura de quadril foi fator de risco para osteoporose. A principal influência sobre o pico de massa óssea de uma mulher, isto é, o máximo de ganho de DMO durante o desenvolvimento do esqueleto e a fase de maturação, é a hereditariedade. A história de fratura em parentes de primeiro grau aumenta o risco para fratura. Em concordância aos nossos resultados, em estudo de metanálise o antecedente familiar de fratura de quadril esteve relacionado à elevada taxa de risco $(\mathrm{RR}=2,27)$ para fraturas osteoporóticas ${ }^{16}$.

Neste estudo, as pacientes foram classificadas em média como sobrepeso (IMC de 25 a $29,9 \mathrm{~kg} / \mathrm{m}^{2}$ ); apenas $5,1 \%$ apresentavam IMC inferior a $21 \mathrm{~kg} / \mathrm{m}^{2}$. Maior IMC mostrou-se importante fator protetor para a doença. Apenas 5\% das pacientes com IMC menor que $20 \mathrm{~kg} / \mathrm{m}^{2}$ apresentavam DMO normal, comparado com $45 \%$ das pacientes com IMC maior que $30 \mathrm{~kg} / \mathrm{m}^{2}$. Baixo peso corporal caracterizado abaixo de $57,7 \mathrm{~kg}$ para mulheres acima de 65 anos ou IMC inferior a $21 \mathrm{~kg} / \mathrm{m}^{2}$, comprovadamente contribuírem para baixa DMO, além do aumento do risco de fratura especialmente em mulheres mais velhas ${ }^{24}$. Dados da literatura justificam esta correlação positiva entre peso corporal e DMO. A maior conversão periférica dos hormônios gonadais em pacientes obesas melhora a manutenção da massa óssea, protegendo contra os efeitos adversos da deficiência estrogênica sobre o esqueleto ${ }^{25}$.

Entre as participantes deste estudo, o tabagismo atual aumentou significativamente o risco para baixa DMO, em concordância com dados da literatura. Em nosso estudo, $35 \%$ das pacientes tabagistas apresentavam osteoporose. Entre as não fumantes, somente $21 \%$ eram acometidas. Quando comparadas a não fumantes, as mulheres tabagistas apresentam menor massa óssea e tendem a perder mais rapidamente a DMO, além de maior risco de fraturas ${ }^{26,27}$. O mecanismo pelo qual o cigarro afeta a massa óssea não é conhecido, entretanto evidências sugerem que ocorra interferência na absorção do cálcio e menor nível sérico do estradiol ${ }^{26}$. Estudo da OMS indicou que a história de tabagismo confere substancial risco futuro para fratura, independentemente da $\mathrm{DMO}^{28}$.

Certamente, exercícios físicos como a caminhada, a corrida e a musculação, interferem positivamente no sistema musculoesquelético por estimularem a osteogênese ${ }^{29,30}$. Além disso, exercícios regulares estão associados com diminuição do risco de fratura, bem como redução de queda devido ao aumento da massa e força muscular ${ }^{31}$. Entretanto, no presente estudo não foi observada associação entre a prática de exercício físico e a DMO. Apenas $27,7 \%$ das participantes referiram prática de exercícios físicos regulares, principalmente caminhadas três vezes na semana, o que possivelmente não repercutiu na DMO, por tratar de exercícios de baixa intensidade e pouca frequência. Estudos indicam que exercícios de resistência com pesos podem ser benéficos ao ganho e manutenção da massa óssea pelo maior estímulo ósseo ${ }^{31}$.

Recente pesquisa epidemiológica denominada Brazilian Osteoporosis Study (BRAZOS) ${ }^{32}$, avaliou os fatores clínicos de risco para fratura osteoporótica em amostra representativa de 150 diferentes cidades em cinco macros regiões brasileiras, um total de 2.420 indivíduos ( $70 \%$ mulheres, $\mathrm{n}=1694)$. Aproximadamente $15 \%$ das mulheres e $13 \%$ dos homens apresentavam fratura por fragilidade óssea. Em mulheres, os principais fatores de risco foram: idade avançada, história familiar de fratura de quadril, menopausa precoce, sedentarismo, alta ingestão de fósforo, baixa qualidade de vida, diabetes mellitus, uso de benzodiazepínicos e queda recorrente. Portanto, intervenções clínicas como abandonar o fumo, estimular atividade física regular e prevenir quedas podem reduzir o risco de fraturas ${ }^{32}$. O mesmo grupo de pesquisadores brasileiros publicou em 2010, novo estudo epidemiológico com 4.332 mulheres na pré e pós-menopausa ${ }^{33}$. Desta vez, além do risco de fratura 
de fragilidade, os fatores de risco foram comparados com dados densitométricos para avaliação do risco para osteoporose. Concluíram que idade avançada, maior tempo de menopausa e antecedente pessoal de fratura de fragilidade, foram fatores de risco para osteoporose. Atividade física regular, elevado IMC, dieta adequada e uso de terapia hormonal foram fatores protetores ${ }^{33}$. Estes dados estão em concordância com o nosso estudo.

A associação de fatores de risco com a medida da DMO é de suma importância, embora sejam poucas as pacientes que têm oportunidade de realizar a densitometria óssea. Consensos sobre osteoporose têm propostos algoritmos para identificar indivíduos de alto risco para fraturas. Nesse sentido, a OMS por meio de metanálise identificou fatores independentes de risco para fratura e a seguir realizou uma análise global envolvendo 12 estudos de coorte, com aproximadamente 250.000 pessoas/ ano, 60.000 pacientes e 5.000 fraturas. A independente contribuição de cada fator de risco foi usada para computar a probabilidade de fratura na ausência de fatores ou na presença de várias combinações ${ }^{13}$. Assim, surgiu um algoritmo denominado fracture risk assessment tool $\left(\mathrm{FRAX}^{\oplus}\right)$, que permite o cálculo computado da probabilidade (\%) de determinado indivíduo, acima de 40 anos, ter uma fratura de quadril e em outros locais (vértebras, úmero distal ou forame proximal) por fragilidade nos próximos 10 anos. Esta ferramenta leva em conta fatores de risco como idade, sexo, peso e altura (IMC), ocorrência prévia de fratura por fragilidade, mãe ou pai com fratura de quadril, tabagismo atual, uso de glicocorticóide, AR, osteoporose secundária, uso de álcool ( $\geq 3$ doses) associado ou não ao valor de escore $\mathrm{T}$ de colo de fêmur na densitometria óssea. Se a paciente não tem densitometria, os fatores clínicos podem ajudar a decidir o tratamento do paciente ${ }^{34}$. O Brasil é um país com dimensões continentais, tem uma população com grande miscigenação e diversidade étnica. Logo, a extrapolação dos dados americanos, europeus ou mesmo de países latinoamericanos é inadvertida por se tratar de uma doença que tem a genética como principal etiologia. Os estudos atuais ainda não permitem a formulação deste algoritmo para a nossa população. Como consequência, a ferramenta FRAX $^{\circledR}$ não pode ser utilizada no Brasil ${ }^{35}$.

Os dados deste estudo podem ser limitados, pois se trata de corte transversal e as participantes reportaram dados referentes a estilo de vida, dieta, antecedentes familiares e pessoais, o que pode ter ocasionado algum viés recordatório. Outra limitação é que a maior parte das mulheres são de baixo nível socioeconômico (2 a 3 salários-mínimos) sem acesso à adequada educação nutricional (dieta rica em cálcio, vitamina $\mathrm{D}$ e proteínas) e à atividade física orientada. A terceira limitação é em relação à população analisada, que foi de pacientes seguidas em ambulatório público específico na assistência à mulher climatérica, em que a TH é rotineiramente indicada no alívio da sintomatologia climatérica. A proporção de usuárias de TH é elevada e a faixa etária predominante é de mulheres nos primeiros anos de pós-menopausa; apenas 5,6\% tinham idade $\geq 65$ anos. Mas é importante ressaltar que as participantes se beneficiam do atendimento especializado onde é investigada a ocorrência de diversas doenças, entre elas a osteoporose.

Em conclusão, entre mulheres na pós-menopausa, a idade, tempo de menopausa, tabagismo e a história materna de fratura foram indicadores clínicos do risco para osteoporose, enquanto que uso de enquanto que o uso de TH e o IMC foram fatores protetores.

\section{Agradecimentos}

À Fundação de Amparo da Pesquisa do Estado de São Paulo (FAPESP) pelo apoio financeiro por meio do auxílio à pesquisa pelo processo no ${ }^{\circ} 2008 / 1378-2$.

\section{Referências}

1. Instituto Brasileiro de Geografia e Estatística [homepage on the Internet]. Censo 2010 [cited 2011 Mar 10]. Available from: http://www.ibge.gov.br/censo2010/.

2. Cumings SR, Melton L. Epidemiology and outcomes of osteoporotics fractures. Lancet. 2002;359(9319):1761-7.

3. Kanis JA. Assessment of fracture risk and its application to screening for postmenopausal osteoporosis: synopsis of a WHO report. WHO Study Group. Osteoporos Int. 1994;4(6):368-81.

4. Riggs BL, Khosla S, Melton L 3rd. A unitary model for involutional osteoporosis: estrogen deficiency causes both type I e type II osteoporosis in postmenopausal women and contributes to bone loss in aging men. J Bone Miner Res. 1998; 13(5):763-73.

5. Reginster JY, Burlet N. Osteoporosis: a still increasing prevalence. Bone. 2006;38(2 Suppl 1):S4-9.
6. Brasil. Ministério da Saúde [homepage on the Internet]. Saúde do idoso: apresentação. [cited 2011 Mar 15]. Available from: http://portal.saude.gov.br/portal/saude/visualizar_texto. cfm? idtxt=26466.

7. WHO Scientific Group on the assessment of osteoporosis at primary health care level. Summary Meeting Report [homepage on the Internet]; 2004 May 5-7; Brussels, Belgium [cited 2011 Mar 15]. Available from: http://www.who.int/chp/topics/ Osteoporosis.pdf.

8. Management of osteoporosis in postmenopausal women: 2010 position statement of the North American Menopause Society Menopause. Menopause. 2010;17(1):25-54.

9. Siris ES, Miller PD, Barrett-Connor E, Faulkner KG, Wehren $L E$, Abbott $T A$, et al. Identification and fracture outcomes of 
undiagnosed low bone mineral density in postmenopausal women: results from the National Osteoporosis Risk Assessment. JAMA. 2001;286(22):2815-22.

10. Silverman SL. Selecting patients for osteoporosis therapy. Curr Osteoporos Rep. 2006;4(3):91-5.

11. Kanis JA, Johnell O, Oden A, Dawson A, De Laet C, Jonsson B. Ten year probabilities of osteoporotic fractures according to $B M D$ and diagnostic thresholds. Osteoporos Int. 2001;12(12):989-95

12. Kanis JA, Borgstrom F, De Laet $C$, Johansson $H$, Johnell $O$, Jonsson $B$, et al. Assessment of fracture risk. Osteoporos Int. 2005; 16(6):581-9.

13. Kanis JA, Oden A, Johnell O, Johansson H, De Laet C, Brown J, et al. The use of clinical risk factors enhances the performance of $B M D$ in the prediction of hip and osteoporotic fractures in men and women. Osteoporos Int. 2007;18(8): 1033-46.

14. Doherty DA, Sanders KM, Kotowicz MA, Prince RL. Lifetime and fiveyear age specific risks of first and subsequent osteoporotic fractures in postmenopausal women. Osteoporos Int. 2001;12(1):16-23.

15. Klotzbuecher CM, Ross PD, Landsman PB, Abbott TA 3rd, Berger $M$. Patients with prior fractures have an increased risk of future fractures: a summary of the literature and statistical synthesis. J Bone Miner Res. 2000; 15(4):721-39.

16. Kanis JA, Johansson $H$, Oden A, Johnell O, De Laet C, Eisman JA, et al. A family history of fracture and fracture risk: a meta-analysis. Bone. 2004;35(5): 1029-37.

17. National Osteoporosis Foundation [homepage on the Internet]. Clinician's guide to prevention and treatment of osteoporosis. Washington (DC): NOF; 2010 [cited 2011 Mar 15]. Available from : http://www.nof.org/sites/default/files/pdfs/NOF_ ClinicianGuide2009_v7.pdf.

18. Recker R, Lappe J, Davies K, Heaney R. Characterization of perimenopausal bone loss: a prospective study. J Bone Miner Res. 2000; 15(10): 1965-73

19. Demir B, Haberal A, Geyik P, Baskan B, Ozturkoglu E, Karacay $O$, et al. Identification of risk factors for osteoporosis among postmenpausal women. Maturitas. 2008;60(3-4):253-6.

20. Khosla S. Update on estrogens and the skeleton. J Clin Endocrinol Metab. 2010;95(8):3569-77.

21. Wells G, Tugwell P, Shea B, Guyatt G, Peterson J, Zytaruk N, et al. Meta-analyses of therapies for postmenopausal osteoporosis. V. Meta-analysis of the efficacy of hormone replacement therapy in treating and preventing osteoporosis in postmenopausal women. Endocr Rev. 2002;23(4):529-39.

22. Cauley JA, Robbins J, Chen Z, Cummings SR, Jackson RD, LaCroix $A Z$, et al, for the Women's Health Initiative Investigators. Effects of estrogen plus progestin on risk of fracture and bone mineral density: the Women's Health Initiative randomized trial. JAMA. 2003;290(13): 1729-38.

23. Anderson GL, Limacher M, Assaf AR, Bassford T, Beresford SA, Black $\mathrm{H}$, et al. Effects of conjugated equine estrogen in postmenopausal women with hysterectomy: the Women's Health Initiative randomized controlled trial. JAMA. 2004;291(14):1701-12.

24. De Laet C, Kanis JA, Oden A, Johanson H, Johnell O, Delmas $P$, et al. Body mass index as a predictor of fracture risk: a metaanalysis. Osteoporos Int. 2005;16(1 1):1330-8.

25. Heiss CJ, Sanborn CF, Nichols DL, Bonnick SL, Alford BB. Associations of body fat distribution, circulating sex hormones, and bone density in postmenopausal women. J Clin Endocrinol Metab. 1995;80(5):1591-6.

26. Rapuri PB, Gallagher JC, Balhorn KE, Ryschon KL. Smoking and bone metabolism in elderly women. Bone. 2000;27(3):429-36.

27. Baron JA, Farahmand BY, Weiderpass E, Michaëlsson K, Alberts A, Persson I, et al. Cigarrete smoking, alcohol consumption, and risk for hip fracture in women. Arch Intern Med. 2001;161 (7):983-8.

28. Kanis JA, Johnell $O$, Oden A, Johansson $H$, De Laet $C$, Eisman $\mathrm{JA}$, et al. Smoking and fracture risk: a meta-analysis. Osteoporos Int. 2005; 16(2): 155-62.

29. Kohrt WM, Bloomfield SA, Little KD, Nelson ME, Yingling VR; American College of Sports Medicine position stand: physical activity and bone health. Med Sci Sports Exerc. 2004;36(1 1):1985-96.

30. Gjesdal CG, Halse JI, Eide GE, Brun JG, Tell GS. Impact of lean mass and fat mass on bone mineral density: The Hordaland Health Study. Maturitas. 2008;59(2):191-200.

31. Carter ND, Kannus $P$, Khan KM. Exercise in the prevention of falls in older people: a systematic literature review examining the rationale and the evidence. Sports Med. 2001;31(6):427-38.

32. Pinheiro MM, Ciconelli RM, Martini LA, Ferraz MB. Clinical risk factors for osteoporotic fractures in Brazilian women and men: the Brazilian Osteoporosis Study (BRAZOS). Osteoporos Int. 2009;20(3):399-408.

33. Pinheiro MM, Reis Neto ET, Machado FS, Omura F, Yang JH, Szejnfeld J, et al. Risk factors for osteoporotic fractures and low bone density in pre e postmonopausal women. Rev Saúde Pública, 2010;44(3):479-85.

34. Kanis JA, Johnell $O$, Oden $A$, Johansson $H, M c C l o s k e y ~ E$. FRAX ${ }^{T M}$ and the assessment of fracture probability in men and women from the UK. Osteoporos Int. 2008; 19(4):385-97.

35. Pinheiro MM, Camargos BM, Borba VZC, Lazaretti-Castro M. Frax TM: construindo uma ideia para o Brasil. Arq Bras Endocrinol Metab. 2009;53(6):783-90. 